Erschienen in: Stickel, Gerhard (Hrsg.): Stilfragen. - Berlin, New York: de Gruyter, 1995. S. 115-127. (Institut für deutsche Sprache. Jahrbuch

1994)

\title{
JOHANNES ANDEREGG
}

\section{Stil und Stilbegriff in der neueren Literaturwissenschaft}

1.

Die Antwort auf die Frage, die sich im Titel versteckt, läßt sich mit wenigen Worten umreißen: In der Literaturwissenschaft ist es still geworden um Stil und Stilbegriff. Alles deutet darauf hin, daB Stilbegriff und Stiluntersuchung ihre große und gute Zeit hinter sich haben - nämlich die Zeit der sogenannten Werkinterpretation. Wenn die Werkinterpretation sich durch besondere Textnähe auszeichnete, so ist dies in erster Linie der damals dominierenden Frage nach dem Stil zuzuschreiben. Und die Frage nach dem Stil war es auch, die den Zugang zum 'Kern der Sache' zu gewähren versprach, zu dem nämlich, was den Text zum Kunstwerk zu machen schien, zu seinem ästhetischen Wert. Grundlegend war dabei die Vorstellung der Einstimmigkeit des literarischen Texts - ein spätes Kind des klassischen Ideals von der Einheit in der Vielfalt -, und die Stiluntersuchung galt als das Instrument, mit dem sich die Einstimmigkeit erfassen lieb.

Zum Ausbau der Stiltheorie hat die Werkinterpretation wenig beigetragen, und die damaligen methodischen Überlegungen zur Stiluntersuchung nehmen sich aus heutiger Sicht erschreckend bescheiden aus. Was aber die Praxis der Stiluntersuchung betrifft, so sind im Rahmen der Werkinterpretation Arbeiten von hohem, ja höchstem Rang vorgelegt worden. $\mathrm{Zu}$ erinnern wäre etwa an Richard Alewyns Beitrag über Eichendorff, an Walther Rehms Untersuchung von Rumohrs Kochkunst, an Emil Staigers programmatische Aufsätze zu Kleist oder Brentano und - um auch einen Vertreter jüngerer Generationen zu nennen - an Albrecht Schönes Analyse von Musils Konjunktivgebrauch. ${ }^{1}$ Diese und zahlreiche andere auf Aspekte des Stils ausgerichteten Arbeiten haben

1 Alewyn, Richard (1957): Eine Landschaft Eichendorfs. In: Euphorion 51, S. 42-60. Wiederabgedruckt in Stöcklein, Paul (Hg.) (1966): Eichendorff heute. Stimmen der Forschung. Mit einer Bibliographie. Darmstadt.

Rehm, Walther (1964): Rumohrs Geist der Kochkunst und der Geist der Goethezeit. In: ders.: Späte Studien. Bern. S. 97-121.

Staiger, Emil (1957): Kleist, Das Bettelweib von Locarno. In: ders.: Meisterwerke deutscher Sprache. Aus dem 19. Jahrhundert. Zürich. S. 100-117. Staiger, Emil (1953): Die Zeit als Einbildungskraft des Dichters. Untersuchungen zu Gedichten von Brentano, Goethe und Keller. Zürich.

Schöne, Albrecht (1961): Zum Gebrauch des Konjunktivs bei Robert Musil. In: Euphorion 55, S. 196-220. 
sich der Geschichte der Germanistik eingeschrieben und sind für den am Text interessierten Leser noch heute von Bedeutung.

\section{2.}

In den sechziger und siebziger Jahren wuchs das Bedürfnis, die Beschäftigung mit Texten als wissenschaftlich auszuweisen, und das heißt auch: sie zu objektivieren. Die Forderung nach einem kritischen MethodenbewuBtsein, die Frage nach der anzuwendenden Methode, der Wunsch nach einer literaturwissenschaftlichen Terminologie oder doch mindestens nach begrifflicher Klärung, und nicht zuletzt die damals weit verbreitete Auffassung, es bedürfe der Umgang mit Literatur generell der Legitimation und der reflektierenden Absicherung, führten zu einer bis dahin unüblichen Theoretisierung der Literaturwissenschaft.

Je mehr die Theorie an Boden gewann, um so mehr verlor die der Theorie wenig geneigte Werkinterpretation an Bedeutung. Das Ansehen des Stilbegriffs aber blieb vorerst unangetastet. Die literaturwissenschaftliche Praxis hatte seine zentrale Bedeutung in vielfaltiger Weise bestätigt; unbezweifelbar schien es, daß Stilbegriff und Stiluntersuchung bei der Annäherung an Literatur Schlüsselfunktionen hatten. Vielerorts scheint der Stilbegriff damals noch als das eigentliche Rückgrat der Literaturwissenschaft gegolten zu haben; an ihm mußte sich deshalb ihre Wissenschaftlichkeit erweisen. So galt es denn, nachzuholen, was man bisher versäumt hatte: Der Begriff des Stils mußte definiert und in einen Theorierahmen eingefügt werden; die Stiluntersuchung bedurfte der methodologischen Grundlegung.

Aus dem Bedürfnis heraus, den Begriff des Stils und seinen Gebrauch zu klären und festzuschreiben, entwickelte sich bald ein lebhafter Definitionsstreit, und Stiltheorien unterschiedlichster Art traten miteinander in Konkurrenz. Traditionalisten versprachen sich, nicht nur terminologisch unbekümmert, von der Stiluntersuchung den Zugang zur "Gemüthaftigkeit" des Werks" ${ }^{2}$, man bezog den Stilbegriff, den Spuren Ballys folgend ${ }^{3}$, auf den „affektiven Gehalt” von Sprache, und auch Buffons "Le style est l'homme même" erwies sich als revitalisierbar. Bei anderen Definitionsversuchen standen neuere, insbesondere linguistische Entwicklungen oder gerade aktuelle Begriffe Pate: So ließ sich beispielsweise der Gegensatz von Denotation und Konnotation stiltheoretisch

2 Vgl. Seidler, Herbert (1963): Allgemeine Stilistik. 2. Auflage. Göttingen.

3 Bally, Charles (1909): Traité de Stylistique Française I/II. Seconde Edition. Heidelberg/Paris. 
fruchtbar machen. Mit der Bestimmung von Stil als einer Deviation von der Norm kamen frühere theoretische Versuche, insbesondere des Formalismus, zu neuen (und diesmal etwas fragwürdigen) Ehren. Andere liefen sich von Erfahrungen im Bereich der Stillehre leiten und propagierten - unterstützt wohl auch durch damals neuere Entwicklungen in der Grammatiktheorie - einen Stilbegriff, der sich am Prinzip der Wahl orientierte.

Freilich wurde nach kurzer Zeit deutlich, daB mit Einigkeit in der Stilfrage nicht zu rechnen sein würde, die (bald einmal verzweifelten) Anstrengungen, zu einem verbindlichen Stilbegriff zu kommen, erwiesen sich als erfolglos. Der ursprüngliche Optimismus begann angesichts der Vielzahl von Stilbegriffen zu schwinden, und Irritation machte sich breit: Jeder Versuch, das Begriffswirrwarr doch noch zu beseitigen und ihm endlich die stichfeste, gültige Definition entgegenzusetzen, vergrößerte nur das heillose Neben- und Durcheinander.

Gründe für das Scheitern der literaturwissenschaftlichen Bemühungen um den Stilbegriff lagen auf der Hand. Es ließ sich und es läßt sich ohne großen Aufwand zeigen, daB einige der Definitionsversuche zu eng, $\mathrm{daB}$ andere zu weit waren, daB widersprüchlich oder tautologisch argumentiert wurde. Wichtiger ist im hier gegebenen Zusammenhang aber die Erfahrung, daB die stiltheoretische Auseinandersetzung bei allen Fragwürdigkeiten im einzelnen den Sinn für die differenzierte Textbetrachtung zu schärfen vermochte und wichtige Aspekte des Rezeptionsvorgangs bewubt machte. Und auch dies verdient festgehalten zu werden: Zumindest für einige Zeit war die Frage nach dem Stilbegriff Anlaß für einen freundlichen Brückenschlag $\mathbf{z w i s c h e n ~ L i t e r a t u r w i s s e n s c h a f t ~ u n d ~}$ Linguistik.

\section{3.}

Dem stiltheoretischen Sturm der sechziger und siebziger Jahre ist mittlerweile, jedenfalls in der Literaturwissenschaft, die Flaute gefolgt. Wolfgang Kayser hat 1948 in seinem damals grundlegenden und über die Grenzen des deutschen Sprachraums hinaus bekannten Buch „Das sprachliche Kunstwerk" ${ }^{4}$ eines von zehn Kapiteln dem Stil gewidmet. Fünfundzwanzig Jahre später, 1973, finden sich in "Grundzüge der Literatur- und Sprachwissenschaft" 5 neben Ausführungen zu Rheto-

4 Kayser, Wolfgang (1948): Das sprachliche Kunstwerk. Eine Einführung in die Literaturwissenschaft. Bern.

5 Arnold, Heinz Ludwig/Sinemus, Volker (Hg.) (1973): Grundzūge der Literatur- und Sprachwissenschaft. Band I. Literaturwissenschaft. München. 
rik und Poetik immerhin noch zwanzig Seiten zu Stilbegriff und Stiltheorie. In Eagletons "Einführung in die Literaturtheorie”, seit 1988 in deutscher Übersetzung vorliegend, ist dagegen über Stil oder Stiltheorie kein Wort zu finden, und die "Critical Terms for Literary Study" 7 von F. Lentricchia und Th. McLaughlin von 1990 führen zwar 'Structure', 'Discourse', 'Rhetoric' und 'Gender' auf, aber über den Begriff des Stils schweigt sich der Band aus. Gewiß finden sich auch Gegenbeispiele, so die 1991 erschienene "Einübung in die Literaturwissenschaft" 8 von $\mathrm{H}$. Fricke und R. Zymner; es handelt sich dabei aber bezeichnenderweise nicht eigentlich um eine Theorie der Literatur oder der Literaturwissenschaft, sondern um eine praktische Anleitung für den Umgang mit Texten.

Dem Abgesang auf den Stil ist indes ein deutliches „und dennoch!” entgegenzusetzen. Weder das Versagen der Stiltheorie, noch die wiederholte Versicherung, es gäbe keinen Stil und die Stiluntersuchung sei tot, haben in der Praxis die Stiluntersuchung oder allgemeiner die Frage nach dem Stil verdrängen können. So ist fast unvermeidbar von Stil die Rede, wo immer das Lesen gelehrt wird, und dabei fallt auf, daß auch durchaus ungeklärte, ja widersprüchliche Verwendungen des Stilbegriffs zu brauchbaren Resultaten führen und das Textverständnis fordern können. Auch haben sich offenbar wichtige und wichtigste Autoren gerade ihres Stils wegen in das literarische BewuBtsein eingeschrieben - an Eichendorff und Kleist wäre zu erinnern, an Thomas Mann und an Max Frisch -, und es ist schwer denkbar, daß von solchen Autoren kompetent gehandelt wird, ohne daB Stilistisches zur Sprache käme.

Im Zusammenhang mit typologischen Fragen, deren Wissenschaftlichkeit erstaunlich wenig bezweifelt wird, ist die Stilfrage nach wie vor kaum vermeidbar; wenn es um Zuordnungen zu Strömungen und Epochen geht oder zu unterschiedlichen Phasen eines Gesamtwerks, ist uns das Reden über Stil geläufig und unproblematisch. Und auch dann ist vom Stil die Rede, wenn es gilt, Beziehungen zwischen der Literatur und anderen Künsten zu erhellen.

6 Eagleton, Terry (1988): Einführung in die Literaturtheorie. Stuttgart.

7 Lentricchia, Frank/McLaughlin, Thomas (Hg.) (1990): Critical Terms for Literary Study. Chicago.

8 Fricke, Harald/Zymner, Rüdiger (1991): Einübung in die Literaturwissenschaft: Parodieren geht über Studieren. Paderborn. 
DaB Fragen nach Stil und Stilistischem nach wie vor aktuell und sinnvoll sein können, zeigt mit besonderer Deutlichkeit die gegenwärtige Literaturkritik, die in erstaunlich hohem Maße Stilkritik ist - gleichgültig, ob es sich dabei um das traditionelle Besprechungswesen, um öffentliche Jurydiskussionen oder um Fernsehshows handelt. Allerdings ist es wohl gerade die Neigung der Literaturkritik zur Stilkritik, die manchen Wissenschaftler davon abhält, die Literaturkritik als Wissenschaft zu anerkennen: Dem Reden über Stilistisches fehlt es an Präzision; oft klingt es nicht wissenschaftlich, sondern alltäglich. Und wer wollte es bestreiten: Das Reden über Stilistisches gehört durchaus zu unserem Alltag. Im Alltäglichen - und ganz ohne wissenschaftliche Aspirationen - nehmen wir Stil wahr, werden wir von Stilistischem beeinflußt. Oft gilt beispielsweise schon das erste Blättern in einem neuen Buch dem Stil, und aufgrund stilistischer Eindrücke entscheiden wir uns für oder gegen den Kauf. Flüchtige Lektüre genügt, um einen Text dem Stil des Rokoko oder dem des Sturm und Drang zuzuordnen, und wenn wir einen Text, mit erheblicher Sicherheit, diesem oder jenem Autor zuschreiben, Chandler oder Highsmith, Frisch oder Handke, so tun wir dies aufgrund unserer stilistischen Erfahrung und Wahrnehmung. Auch wenn uns das als Wissenschaftler vielleicht nicht genehm ist: Stilistische Aspekte eines Textes drängen sich unserer Wahrnehmung auf, sprechen uns an oder irritieren uns - lange bevor wir angefangen haben, über den Text nachzudenken und lange bevor wir imstande sind, den Text wissenschaftlich zu befragen.

Weithin unentbehrlich in der Praxis - kaum mehr beachtet in der Theorie: So ließe sich der gegenwärtige Status von Stil und Stilbegriff zusammenfassen. Die folgenden Überlegungen gelten denn auch vor allem diesem Gegensatz zwischen Theorie und Praxis. Wie kommt es, daß wir die Frage nach dem Stil aus unseren theoretischen Überlegungen ausklammern, obwohl wir sie tagtäglich in verschiedensten Zusammenhängen stellen? Wie läßt es sich erklären, daß die Wissenschaft einen Begriff unbeachtet läßt, dessen Aktualität sich immer von neuem zu bestätigen scheint?

4.

Im Feld der Praxis, beim unmittelbaren Umgang mit Literatur ist die Frage nach dem Stil vor allem eine Ordnungs- und Orientierungsfrage. Wer nach dem Stil fragt, will sich in der sprachlichen Vielfalt - sei es eines einzelnen Texts, sei es mehrerer Texte oder Textgruppen - orientieren. 
Er sucht nach Zusammenhängen, bemüht sich, sprachliche Erscheinungen zu bündeln, unterscheidet Verbindendes und Trennendes, um so im Vielgestaltigen eine Ordnung zu entdecken oder herzustellen.

Diese Feststellung ist, bei aller Selbstverständlichkeit, insofern bemerkenswert, als der literarische Text dazu angetan ist, bestehende konventionelle Ordnungen in Frage zu stellen, sie zu verändern oder aufzulösen. So zumindest - als Infragestellung unserer konventionellen Wahrnehmungsordnungen - verstehen wir den literarischen Text, wenn wir ihn in seiner Literarität zu erfassen und ernst zu nehmen trachten. Wenn die Stiluntersuchung des literarischen Texts seiner 'Subversivität' oder seiner Widerborstigkeit Rechnung tragen soll, ist es zumindest unwahrscheinlich, da B sie ihm in einer Systematik, in einer bestehenden Ordnung also, einen eindeutigen Ort zuweisen kann, und es erscheint widersprüchlich oder mindestens fragwürdig, ihm einen solchen Ort überhaupt zuweisen zu wollen. Das heiBt aber, daß die Frage nach dem Stil beim literarischen Text an ihre Grenze kommt. Sie entspringt einem dringenden und legitimen Ordnungs- und Orientierungsbedürfnis, aber eine gesicherte Ordnung läßt sie nicht erwarten, und sie kann sie, wenn sie ihrem Gegenstand gerecht werden will, nicht einmal anbieten wollen. Sie wird durch das, worauf sie gerichtet ist, $\mathbf{z w a r}$ nicht aufgehoben, wohl aber wird sie vom literarischen Text gewissermaßen zurückgeworfen.

Wer sich orientieren will, wer, um sich zu orientieren, nach Ordnungen sucht, ist auf das Vergleichen angewiesen. Dabei versteht es sich von selbst, daß das Ziel nur erreicht werden kann, wenn der Bereich des Vergleichbaren begrenzt ist. Eben diese Voraussetzung ist, wenn es um die stilistische Ordnung im Literarischen geht, nicht gegeben: Das Vergleichskorpus ist weder klar umgrenzt noch läßt es sich objektiv festschreiben. Selbst wenn Kanonbildungen zu gesellschaftlichen Gemeinsamkeiten führen oder kulturell bedingte Verhaltensweisen und Werthaltungen die Wahrnehmungen kanalisieren, bleibt die Tatsache bestehen, daB Leser sich bei ihrem ordnenden Vergleichen auf die je eigene Leseerfahrung beziehen. Oder, um es mit den Worten von Barbara Sandig zu sagen: "Was Stil ist, ist abhängig von den Kenntnissen des Rezipienten." 9 Besonders fällt dabei ins Gewicht, daß das Textkorpus, das vergleichend herangezogen werden kann, ständigem Wachstum ausgesetzt ist. Um das Problem mit einem Beispiel zu verdeutlichen: Der wohlgefügte Satz ist nach James Joyce nicht mehr, was er zuvor war. Das Fortschreiben verändert den Stellenwert des Geschriebenen.

9 In ihrem Vortrag "Tendenzen der linguistischen Stilforschung" am 15.3.1994. 
Das heiBt nun aber, daß die Frage nach dem Stil, insofern sie Ordnungsund Orientierungsfrage ist, immer nur zu einer relativen, zu einer vorläufigen Antwort führen kann. Oder anders und härter: Als Ordnungsfrage bleibt die Frage nach dem Stil im Bereich des Literarischen eine grundsätzlich unerledigte Frage. Nicht, då sie deshalb an Bedeutung verlöre. Im Gegenteil: Weil immer wieder Neues geschieht oder Neues entdeckt wird, werden bisherige Ordnungen fragwürdig, bedürfen wir von neuem der Orientierung. Die Fragwürdigkeit der Antwort hält die Stilfrage am Leben.

Die Legitimation der Frage nach dem Stil - und die Legitimation ihrer Repetition - sind augenfällig: Die Frage nach dem Stil entspricht dem grundlegenden Bedürfnis - wenn man so will: dem anthropologischen Grundbedürfnis -, sich im Vielfältigen zurechtzufinden und den verunsichernden Veränderungen durch jeweils neue Orientierung $z u$ begegnen. Zugleich aber dürfte nun auch deutlich geworden sein, dab die Frage nach dem Stil als eine, zumindest im Literarischen, grundsätzlich unerledigte Frage der Wissenschaft suspekt sein muB; sie grenzt sich jedenfalls aus dem Bereich jener Wissenschaft aus, deren Ziel es ist, erworbenes Wissen systematisch geordnet zu kumulieren, die darum als Resultat gelten läßt, was festgeschrieben werden kann, und deren Stärke darin liegt, daß sie das einmal Erworbene nicht mehr in Frage stellen muB.

5.

Die Frage nach dem Stil ist allerdings nicht nur eine Ordnungs- oder Orientierungsfrage, sondern auch eine Identitäts- oder Identifikationsfrage. Sie zielt auf das je Eigenartige und Einmalige - sei es eines einzelnen Texts, sei es einer Textgruppe, eines Gesamtwerks etwa, einer Gattung oder einer historischen Strömung.

Als Identifikations- und Identitätsfrage gründet die Frage nach dem Stil in unserem Bedürfnis, das Konstante im Wechselnden, im Verschiedenen das Identische zu erkennen. Impetus des Fragens ist stets das identifizierende Wiedererkennenwollen, und die Beschäftigung mit dem Stil ist darum immer Beschäftigung mit Wiederkehrendem, mit Wiedererkennbarem. So gesehen ist die Frage nach dem Stil ein Kind des 18. Jahrhunderts: Dem modernen Geschichts- und Zeitbewußtsein verdankt die Frage nach dem Gleichbleibenden, nach dem Identischen in der Zeit, ihre besondere Aktualität.

Die Frage nach dem Stil ist nun freilich, auch als Identifikationsfrage, nicht eine Frage nach der Substanz oder nach Substanzen; sie zielt nicht auf Materielles, sondern auf die Organisation von Texten, auf das Funk- 
tionieren von Textelementen. Insofern ist es durchaus richtig, wenn in der Polemik gegen Stilbegriff und Stiluntersuchung immer wieder behauptet wurde, daß es den Stil nicht gäbe: Das Prinzip einer Organisation, das Prinzip des Funktionierens ist nicht in dem Sinne zu haben, wie man etwa chemische Substanzen haben kann. Und genau darum geht es bei der Frage nach dem Stil: Um das Prinzipielle. Wer nach dem Stil fragt, ist keineswegs an allem interessiert, was dem Problem des Organisiertseins oder des Funktionierens zugeordnet werden könnte. Auch fragt er nicht wahllos nach allem, was in irgendeiner Weise wiederkehrt. Auf das Wiederkehrende zielt die Frage nach dem Stil, insofern es ihr um das Prinzip geht, dem Eigenart oder Einmaligkeit zuzuschreiben sind.

Insofern die Frage nach dem Stil als Identifikationsfrage derart auf das Prinzipielle, auf die Art und Weise, ausgerichtet ist und also das Gleiche im Verschiedenen, das Allgemeine im Besonderen sucht, führt sie den Fragenden in ein beunruhigendes Spannungsfeld. Um zum Eigenartigen zu kommen, klopft sie das Verschiedene auf ein Gleiches hin ab; sie sucht das Besondere - eben die Eigenart oder die Identität - als ein Allgemeines. Oder anders: Das Allgemeine, nämlich das Prinzipielle, das sie beschreibt, gilt ihr als das Besondere, Eigenartige. Die Stilfrage erweist sich als ein Irritans, denn was sie als Identitätsfrage zu Tage fördert, ist gerade das, was die Identität in Frage stellt: Das Prinzipelle ist nicht nur das Wiederkehrende, sondern grundsätzlich auch das Wiederholbare; das rufen uns Stilzitate und Stilparodien immer wieder in Erinnerung.

Das Prinzipielle, das die Stiluntersuchung zu fassen trachtet, macht den Text oder die Textgruppe zu einer Art Ganzheit: Weil im Verschiedenen das eine Prinzip erkennbar wird, läßt es sich als ein Zusammengehöriges begreifen. Dennoch erfaBt die Stiluntersuchung ihren Gegenstand - den Text oder die Textgruppe - gerade nicht in seiner Ganzheit, und dies nicht nur, weil bei unterschiedlich angelegten Stiluntersuchungen jeweils verschiedene Aspekte ein und desselben Texts beleuchtet werden. Immer ist ein Text noch etwas anderes als das, was er stilistisch ist. Der Text, den ich aufgrund seines Stils einem Autor, einer Zeit, einer Gattung zuweise, ist immer mehr als das, was ihn zum Text dieses Autors, dieser Zeit oder dieser Gattung macht. Die Stilfrage ist darum immer nur eine mögliche Frage neben anderen Fragen, und ihre Relevanz muß sie diesen gegenüber durch ihr Resultat allererst erweisen. Wo wissenschaftliche Disziplin den Ton angibt, wird die Frage nach dem Stil darum kaum Anerkennung finden. Wissenschaftliche Disziplinen pflegen zu disziplinieren: Sie geben die zulässige Fragestellung vor.

Die Frage nach dem Stil führt zu einer Identifizierung, die weder als erschöpfend, noch als abschließend gelten kann; sie ist immer nur 
Annäherung an eine Identität, und aller Antwort ist ein "gerade nicht!" mitgegeben. Den Stil eines Textes bestimmen heißt immer: seine Eigenart fassen und nicht fassen. In der Frage nach dem Stil spiegelt sich, so gesehen, die Fragwürdigkeit jeden Bemühens, identifizierend einer Identität habhaft zu werden. Das mindert nicht die Dringlichkeit der Frage; sie bleibt, aller Fragwürdigkeit zum Trotz, so lange bestehen, als wir wahrnehmend und denkend an der Identität interessiert sind. Aber die Tauglichkeit der Frage nach dem Stil für den Dienst im Rahmen wissenschaftlicher Systematisierung läßt sich füglich bezweifeln.

\section{6.}

Im Bereich des Literarischen ist die Frage nach dem Stil nicht nur eine Orientierungs- und eine Identifikationsfrage, sondern immer auch eine Sinnfrage. Wer nach dem Stil fragt, geht davon aus, daß die Art und Weise des Sprachgebrauchs Bedeutung habe, daB sie Sinn mache. Hinter dem Fragen nach Stil steht also - wenn auch freilich in unterschiedlichem Maße (und zuweilen vielleicht gar nicht) reflektiert - die Annahme, oder besser: die Erfahrung, daB Sprache nicht nur aus Zeichen bestehe, sondern daB der Gebrauch dieser Zeichen, daß die Art und Weise des Zeichengebrauchs selbst wieder zeichenhaft, bedeutungsvoll sei; daß Sprache nicht nur ein Instrument sei, um sich in einer vorfindbaren Welt bzw. über eine vorfindbare Welt zu verständigen, daB in ihr vielmehr jene Welt erst konstituiert werde, die wir für die wirkliche halten, oder daB zumindest durch Sprache die Sichtweise bestimmt werde, in der uns das Wirkliche zugänglich wird.

Auch wenn der Frage nach dem Stil im einzelnen recht unterschiedliche Auffassungen sowohl von Sprache als auch von Wirklichkeit zugrunde liegen mögen: als Sinnfrage geht sie davon aus, daß durch die Art und Weise des Sprachgebrauchs die besondere Welt eines Textes konstituiert oder aktualisiert wird. Nach dem Stil fragen heiBt auch nach jener Welt fragen, die durch die Art und Weise des Sprachgebrauchs hervorgebracht wird. So jedenfalls reden wir aufgrund von stilistischen Wahrnehmungen von Schillers hierarchisch-antithetischer Welt, von Rilkes animalisierter Gegenständlichkeit oder davon, daß Max Frisch die Existenz als eine durch und durch temporale erfahre und darstelle.

Die Frage nach dem Stil ist also eine Frage mit hermeneutischer oder semiotischer Perspektivierung. Sie zielt nicht auf wiederkehrende Indizien, sondern auf wiederkehrende Konstitutionen von Sinn. Dabei ist sie - wie dies Szondi schon 1962 im Blick auf alle philologische Tätigkeit 
gezeigt hat ${ }^{10}$ - eine Frage mit zwei Unbekannten. Es gilt zum einen, die Bedeutung von stilistischen Merkmalen oder Zeichen zu erkennen, es gilt zum anderen, stilistische Merkmale oder Zeichen als solche zu identifizieren, und als Merkmal oder Zeichen läßt sich nur das begreifen, was Bedeutung hat.

Insofern die Frage nach dem Stil nicht einfach auf die Wiederholung von Merkmalen, sondern auf die Repetition oder das Prinzip einer Sinnkonstitution ausgerichtet ist, gilt das Interesse nicht eigentlich dem Bleibenden, sondern dem, was immer wieder neu und mit durchaus verschiedenen Mitteln erwirkt wird. Gerade darin besteht der Reiz der Beschäftigung mit Stil, daß der eine Sinn durch sehr unterschiedliche Phänomene oder Zeichen konstituiert werden kann: Die für einen Text oder eine Textgruppe charakteristische Antithetik kann beispielsweise semantisch begründet sein oder aber durch den Satzbau bewirkt werden; die besondere atmosphärische Gestimmtheit eines anderen Textes läßt sich an einer Stelle auf die Denotationen zurückführen, an einer anderen erwächst sie vielleicht aus dem Konnotativen.

Bei derartigen Unsicherheiten und Offenheiten kann es um das wissenschaftliche Ansehen von Stilbegriff und Stiluntersuchung nicht eben gut stehen; dort jedenfalls nicht, wo die Auffassung herrscht oder nachwirkt, es habe sich die Wissenschaft am Kriterium der Eindeutigkeit zu orientieren. Nicht eben gut sind die Chancen für Stilbegriff und Stiluntersuchung auch dort, wo man zur Annahme neigt, Wissenschaft habe zu wissen, wonach sie fragt oder sucht: Die Frage nach dem Stil zeichnet es aus, da $\beta$ sie frei erkunden will, da $\beta$ sie nicht auf von vornherein bestimmte Phänomene ausgerichtet, sonder gewissermaßen auf alles gefaßt ist - auf Affektives oder Rationales, auf Grammatisches oder Rhetorisches etc. - und mit solcher Offenheit auf die Kreativität im Bereich der Textproduktion antwortet.

Wenig Gefallen finden Stil und Stiluntersuchung schließlich auch dort, wo die Wissenschaft bestimmt, was sie als Wirklichkeit akzeptiert, wo sie von der Absicht geleitet wird, das eine Wirkliche zu erforschen und festzuschreiben: Stilbegriff und Stiluntersuchung verdanken ihre Bedeutung ja gerade der Erfahrung und der Einsicht, daß es die eine Wirklichkeit nicht gibt - historisch gesehen wird das Reden vom Stil denn auch gerade

${ }^{10}$ Szondi, Peter (1967): Über philologische Erkenntnis. In: ders.: HölderlinStudien. Mit einem Traktat über philologische Erkenntnis. Frankfurt/Main. S. 9-34. 
dort aktuell, wo der Glaube an die eine Wirklichkeit schwindet -, daB Wirklichkeiten vielmehr konstituiert werden und daß ihre Konstitution mit der Art und Weise oder dem Prinzip des Sprachgebrauchs zu tun habe. Die Stiluntersuchung zielt nicht auf das Festschreiben einer vermeintlich gültigen oder gar endgültigen Wirklichkeit; vielmehr will sie das Festgeschriebene auf seine ihm eigene Wirklichkeit hin befragen.

Freundlichere Aufnahme von Stilbegriff und Stiluntersuchung wäre dort zu erwarten, wo hermeneutische und semiotische Überlegungen an der Tagesordnung sind. Allerdings treten die hermeneutischen Grundprobleme - daB Verschiedenes die gleiche Bedeutung und Gleiches verschiedene Bedeutung haben kann, daB das Merkmal nicht ohne Bedeutung, die Bedeutung aber nicht ohne das Merkmal zu fassen ist - selten so offen zu Tage, wie bei der Beschäftigung mit Stilistischem oder beim Versuch zu beschreiben, was der Begriff des Stils meint und was die Stiluntersuchung leistet; das mag zumindest eine Erklärung dafür sein, daß sich von Stilfragen, vom Nachdenken über Stil und Stilbegriff, manch einer irritieren läßt, dem sonst hermeneutisches Verstehen geläufige Praxis ist.

7.

Bestrebungen, sich im Vielfältigen zu orientieren und im Wechselnden das Konstante zu identifizieren, und Fragen nach dem Sinn von Ordnungen und Konstanten sind in der literaturwissenschaftlichen Praxis nach wie vor häufig mit dem Begriff des Stils verbunden. Stil und Stilbegriff führen hier deshalb ein $z$ war nicht aufregendes aber auch ein nicht ernstlich bedrohtes Leben.

In der Literaturtheorie wirkt dagegen offenbar das Scheitern vieler stiltheoretischer Versuche nach. Dementsprechend gering ist die Bereitschaft, das Problem Stil erneut aufzugreifen. Nur selten werden Versuche gemacht, das Scheitern im Bereich der Stiltheorie (als ein möglicherweise notwendiges) zu begreifen ${ }^{11}$, oder dem Thema aus umfassenderen $\mathrm{Zu}$ sammenhängen heraus neue Erkenntnisse abzugewinnen. ${ }^{12}$ Auch stehen

11 Z.B. Anderegg, Johannes (1977): Literaturwissenschaftliche Stiltheorie. Göttingen.

12 So immerhin Gumbrecht, Hans Ulrich/Pfeiffer, K. Ludwig (Hg.) (1986): Stil. Geschich ten und Funktionen eines kulturwissenschaftlichen Diskurselements. Frankfurt/Main. Und Molinié, G./Cahné, P. (Hg.) (1994): Qu'est-ce que le style? Paris. 
Teile der Literaturtheorie noch immer unter dem Einfluß von Vorstellungen 'harter' Wissenschaftlichkeit; ${ }^{13}$ da ist es denn nicht erstaunlich, daß die Beschäftigung mit Stil und Stilbegriff eine Randerscheinung bleibt. Zwar ließe sich ein Begriff von Stil bei entsprechend einschränkender Definition in Theorien unterschiedlichster Art einbauen, doch verlöre er dabei gerade das, was ihn reizvoll, was ihn zu einer Herausforderung macht. BeläBt man ihn aber in seiner irritierenden Offenheit, bringt er gerade jene Unberechenbarkeiten von Literatur ans Licht (etwa die NichtPrädiktabilität des Kreativen), durch die die Kohärenz von Theorien gesprengt zu werden droht; auch spiegeln sich in ihm jene grundsätzlichen Fragwürdigkeiten des menschlichen Begreifens (schon die Geschichtlichkeit des Verstehens darf dazu gezählt werden), die eine 'harte' Wissenschaft eliminieren möchte.

Es wäre freilich ungerecht und falsch anzunehmen, die Literaturwissenschaft oder auch nur die Literaturtheorie weiche derartigen Problemen oder Fragwürdigkeiten grundsätzlich aus. Neuere Entwicklungen machen diese vielmehr zu ihrem eigentlichen Thema. Für Stil und Stilbegriff scheint der Boden aber auch hier nicht günstig zu sein. Wenn im Poststrukturalismus davon ausgegangen wird, daB Sprache nicht ein mehr oder weniger festes System sei, sondern ganz und gar prozeßhaft gesehen werden müsse, ist die Frage nach Stil oder Stilbegriff obsolet, weil sie ja auf die Art und Weise des Umgangs mit eben jenem System zielt, dessen Existenz oder Relevanz bestritten wird. Und wenn die These vertreten wird, es sei $z$ wischen Literatur und dem Reden über Literatur nicht grundsätzlich zu unterscheiden, es seien Texte überhaupt nicht als Entitäten zu betrachten sondern als Fragmente, als Teile einer eigentlich grenzenlosen Vernetzung, so verliert die Frage nach dem Stil ihren Sinn, denn sie setzt, wie unterschiedlich auch immer Stil im einzelnen definiert wird, die Begrenzung ihres Gegenstandes als sinnvoll voraus.

Gewiß sind bei anderer Ausgangslage und in anderen Zusammenhängen stilistische Phänomene nach wie vor von Bedeutung. Aber wo die Literaturwissenschaft ihre Modernität oder Aufgeschlossenheit zum Ausdruck bringen will, vermeidet sie Begriffe, mit denen jene Geschichte zu Wort kommt, die sie überwunden haben will, und in neuen Diskurstypen haben wo nicht neue, so doch unverbrauchtere oder unbelastetere Begriffe das Sagen. So grüBt aus benachbarten Gebieten ein weitläufiger Verwandter

13 Z.B. Fricke, Harald (1991): Literatur und Literaturwissenschaft. Beiträge zu Grundfragen einer verunsicherten Disziplin. Paderborn. 
des guten alten Stils, das 'Styling'; allen Anfechtungen zum Trotz hat sich bisher die 'Struktur' zu halten vermocht; 'Diskurs' und 'Design' sind - wiewohl auch nicht mehr ganz, was sie einmal waren - in aller Munde; Neues verspricht die 'Signatur'; und wer an Stilistischem interessiert ist, aber nicht vom Stil eines Textes, eines Autors oder einer Epoche reden mag, findet Zuflucht bei einem Allerweltswort: er spricht von ihrer 'Sprache'. Damit ist zwar nicht viel gewonnen, aber auch noch nicht viel verloren. 